\title{
Trends of Industry Payments in Neurology Subspecialties
}

Krishna Nalleballe ${ }^{1}$, Karthika Durga Veerapaneni ${ }^{2}$, Yohei Harada ${ }^{2}$, Poornachand Veerapaneni ${ }^{2}$, Narenraj Arulprakash ${ }^{2}$, Jose R. Lopez-Castellanos ${ }^{2}$, Sen Sheng ${ }^{2}$, Julia Rowen ${ }^{3}$, Vasuki Dandu ${ }^{4}$, Sanjeeva Onteddu ${ }^{1}$, Suman Siddamreddy ${ }^{5}$

1. Neurology/Stroke, University of Arkansas for Medical Sciences (UAMS), Little Rock, USA 2. Neurology, University of Arkansas for Medical Sciences (UAMS), Little Rock, USA 3. Pharmacy, University of Arkansas for Medical Sciences (UAMS), Little Rock, USA 4. Neurology, Baptist Health Medical Center, Little Rock, USA 5. Internal Medicine, Baptist Health Medical Center, Little Rock, USA

Corresponding author: Suman Siddamreddy, siddam.suman@gmail.com

\section{Abstract \\ Background}

Open Payments is a national disclosure program to promote transparency by the public disclosure of financial relationships between the pharmaceutical and medical device industries and physicians.

\section{Objective}

To explore payments from the industry to physicians in various neurology subspecialties.

\section{Methods}

Open Payments Program (OPP) data (https://openpaymentsdata.cms.gov) on industry-to-physician payments for the years 2014-2018 were extracted for general neurology, neuromuscular, neurophysiology, and vascular neurology. The data were then analyzed to explore trends in payments for various subspecialties and to identify the possible factors underlying these trends.

\section{Results}

Overall, industry-to-physician payments for neurology subspecialties increased by 16\% from 2014 to 2018. The introduction of newer drugs in a subspecialty was likely the driving factor for higher industry payments. Nearly half of the total industry-to-physician payments were for the subspecialty of multiple sclerosis (MS)/Neuroimmunology; this coincided with Aubagio and Copaxone being the top two medications associated with the highest industry payments in 2014, Aubagio, and Lemtrada in 2018. A significant increase in spending percentages for headache, neuromuscular disorders, and movement disorders was observed while a relative decrease in the payments for MS/neuroimmunology and epilepsy was identified;

Received 07/07/2020

these trends coincide with the introduction of new drugs such as Aimovig, Neuplazid, Nusinersen, and Austedo for headache, neuromuscular and movement disorders.

\section{Conclusions}

From 2014 to 2018, the total industry-to-physician payments for neurology subspecialties increased while the distribution of industry-to-physician payments for various neurology subspecialties showed notable changes. The introduction of newer medications in a subspecialty coincided with higher industry payments. Identification of these trends and potential motives of the industry spending is critical to address any potential physician bias in prescribing medications.

Categories: Neurology, Healthcare Technology, Epidemiology/Public Health

Keywords: health economics, industry payment, open payment program, disparities, neurologist

\section{Introduction}

Financial transactions between the industry and physicians introduce potential conflicts of interest [1-2], which may translate to patient care [3]. To improve transparency, the Sunshine Act, implemented in 2010, mandated the disclosure of industry payments to physicians [4]. Industry payment trends have since been studied for several specialties [5-10]; however, the literature on trends of payments for neurological subspecialties is lacking [11]. We aimed to explore payments from pharmaceutical and device manufacturing companies to various subspecialties of neurology.

\section{Materials And Methods}

The Open Payments Program (OPP) data (https://openpaymentsdata.cms.gov) on industry-to-physician 


\section{Cureus}

payments for the years 2014-2018 were extracted for general neurology, neuromuscular, neurophysiology, and vascular neurology. Subspecialty payments data for 2014-2018 were combined into a single dataset and the variable 'name of associated covered drug or biological' was used to sort the combined dataset. Type of 'drug or biological' for each data point was then studied to ascertain the subspecialty; for example, Tysabri or Tecfidera would suggest the 'multiple sclerosis/neuroimmunology' subspecialty while Vimpat or Aptiom would belong to the 'epilepsy' subspecialty. In the 'associated covered drug or biological' field, we excluded missing data (comprising approximately 3\%) and drugs or biologicals with a frequency of fewer than 50 times (around 1\%). All payment categories were analyzed, including food, travel, research, education, and consulting fees. All data analysis was conducted in SAS version 9.4 (SAS Institute, Cary, NC).

\section{Results}

In 2014, industry-to-physician payments for all neurology subspecialties in the category of drugs and devices were 64 million USD. The three neurology subspecialties receiving the most industry payments were: multiple sclerosis/neuroimmunology (57.1\% of total payments), movement disorders (14.7\%), and epilepsy (14.3\%) (Table 1).

\begin{tabular}{|c|c|c|}
\hline Specialty & 2014 (million USD (\%)) & 2018 (million USD (\%)) \\
\hline MS/Neuroimmunology & 36.5 (57.1\%) & $35.1(46.8 \%)$ \\
\hline Movement & $9.6(14.7 \%)$ & $13.6(18.1 \%)$ \\
\hline Epilepsy & $9.0(14.3 \%)$ & $6.8(9.0 \%)$ \\
\hline Headache & $6.6(10.2 \%)$ & $14.7(19.6 \%)$ \\
\hline Stroke & $2.1(3.4 \%)$ & $2.3(3.1 \%)$ \\
\hline Neuromuscular & $1.3(0.2 \%)$ & $2.5(3.4 \%)$ \\
\hline Total & 64 & 75 \\
\hline
\end{tabular}

TABLE 1: Industry payments by specialty in 2014 and 2018

MS - Multiple sclerosis; USD - United States dollars

Of the top 10 medications, seven drugs were for multiple sclerosis, and one each was for movement disorders, headache, and epilepsy (Table 2).

\begin{tabular}{|c|c|c|c|c|c|}
\hline 2014 & Specialty & Drug & 2018 & Specialty & Drug \\
\hline 1 & MS/Neuroimmunology & Aubagio & 1 & MS/Neuroimmunology & Aubagio \\
\hline 2 & MS/Neuroimmunology & Copaxone & 2 & MS/Neuroimmunology & Lemtrada \\
\hline 3 & Movement Disorders & Azilect & 3 & Headache and Pain & Aimovig \\
\hline 4 & MS/Neuroimmunology & Tecfidera & 4 & MS/Neuroimmunology & Tysabri \\
\hline 5 & Headache and Pain & Botox & 5 & MS/Neuroimmunology & Ocrevus \\
\hline 6 & MS/Neuroimmunology & Tysabri & 6 & MS/Neuroimmunology & Tecfidera \\
\hline 7 & MS/Neuroimmunology & Plegridy & 7 & Movement Disorders & Nuplazid \\
\hline 8 & MS/Neuroimmunology & Gilenya & 8 & Movement Disorders & Austedo \\
\hline 9 & MS/Neuroimmunology & Ampyra & 9 & Headache and Pain & Ajovy \\
\hline 10 & Epilepsy & Aptiom & 10 & Epilepsy & Aptiom \\
\hline
\end{tabular}

TABLE 2: Top 10 drugs: 2014 and 2018

MS - Multiple sclerosis 
By 2018 , the industry-to-physician payments increased by $16 \%$ to a total of 75 million USD. The three subspecialties receiving the most industry payments in 2018 were: multiple sclerosis/neuroimmunology (46.8\% of total payments), headache (19.6\%), and movement disorders (18.1\%). Of the top 10 medications, five drugs were for multiple sclerosis, two each for headache and movement disorders, and one was for epilepsy (Table 2). From 2014 to 2018, there were notable changes in the subspecialty distribution of these industry-to-physician payments (Table 1). For example, payments increased for medications related to headache (from $10.2 \%$ in 2014 to $19.6 \%$ in 2018), neuromuscular disorders (from 0.2 to $3.4 \%$ ), and movement disorders ( $14.7 \%$ to $18.1 \%$ ), while the payments decreased for medications related to multiple sclerosis/neuroimmunology (from $57.1 \%$ to $46.8 \%$ ) and epilepsy (from $14.3 \%$ to $9 \%$ ) and remained stable for stroke-related medications (3.4\% to $3.1 \%$ ).

\section{Discussion}

In this study, we used publicly available databases to explore and report payments from industry to various neurology subspecialties between 2014 and 2018.

There was a significant increase in the total payment to neurology from 2014 to 2018 by $16 \%$. Among them, industry payments for movement disorders, headache, and neuromuscular were increased. Especially, headache and neuromuscular had a substantial increase in these four years. It is consistent with the fact that headache drugs took the third and ninth place of the highest-paid drugs in 2018. There had been no headache medications approved by the Food and Drug Administration (FDA) from 2014 until 2018 when two new drugs (Fremanezumab and Erenumab) came to market (https://www.fda.gov/drugs/new-drugs-fdacders-new-molecular-entities-and-new-therapeutic-biological-products/novel-drug-approvals-2018) (Table 3). 


\section{Cureus}

\begin{tabular}{|c|c|c|c|}
\hline Year & Drug Name & Brand name & Indication \\
\hline \multirow[t]{3}{*}{2014} & Droxidopa & Northera & Neuromuscular \\
\hline & Florbetaben & Neuraceq & Dementia \\
\hline & Peginterferon beta 1a & Plegridy & MS/Neuroimmunology \\
\hline 2015 & Idarucizumab & Praxbind & Stroke \\
\hline \multirow[t]{5}{*}{2016} & Brivaracetam & Briviact & Epilepsy \\
\hline & Pimavanserin & Nuplazid & Movement \\
\hline & Daclizumab & Zinbryta & MS/Neuroimmunology \\
\hline & Eteplirsen & Exondys 51 & Neuromuscular \\
\hline & Nusinersen & Spinraza & Neuromuscular \\
\hline \multirow[t]{6}{*}{2017} & Edaravone & Radicava & Neuromuscular \\
\hline & Valbenazine & Ingrezza & Movement \\
\hline & Deutetrabenazine & Austedo & Movement \\
\hline & Ocrelizumab & Ocrevus & MS \\
\hline & Safinamide & Xadago & Movement \\
\hline & Deflazacort & Emflaza & Neuromuscular \\
\hline \multirow{8}{*}{2018} & Amifampridine & Firdapse & Neuromuscular \\
\hline & Inotersen & Tegsedi & Neuromuscular \\
\hline & Fremanezumab & Ajovy & Headache \\
\hline & Stiripentol & Diacomit & Epilepsy \\
\hline & Migalastat & Galafold & Neuromuscular \\
\hline & Patisiran & Onpattro & Neuromuscular \\
\hline & Cannabidiol & Epidioloex & Epilepsy \\
\hline & Erenumab & Aimovig & Headache \\
\hline
\end{tabular}

TABLE 3: FDA approved drugs 2014-2018

MS - Multiple sclerosis; FDA - Food and Drug Administration

Similarly, there have been many breakthrough advances in the neuromuscular disease field such as Nusinersen for spinal muscular atrophy. According to the FDA data, among 23 newly approved drugs with a neurological indication between 2014 and 2018, nine of them were indicated for neuromuscular disorders. It is also reported that there are currently nearly 200 products in the therapeutic pipeline for neuromuscular disorders and we presume the growth of this field will continue. On the other hand, there is a slight reduction in MS/neuroimmunology, and epilepsy in four years, although six out of the 10 highest paid drug in 2018 were therapies for MS/neuroimmunology. This could be related to the fact that there were no newly FDA-approved MS/neuroimmunology drugs and only one epilepsy drug (Cannabidiol) in 2018. Given that the industry payment for each drug includes the fee for food, travel, research, education, and consulting, the newly approved drug would likely to be received more investment to increase awareness among neurologists. Further studies are needed to evaluate if there is any potential for influence on thought leaders in the field, as has been published before, along with the focus on educational components for newly approved medications [12-14].

\section{Conclusions}

From 2014 through 2018, the distribution of industry-to-physician payments for various neurology subspecialties showed notable changes. Payments to the subspecialties of headache, neuromuscular disorders, and movement disorders increased, likely related to the introduction of newer medications in 
these fields. Physician education and knowledge of the trends and potential motives of industry spending is critical to address any potential bias in prescribing medications when alternatives may be available.

\section{Additional Information \\ Disclosures}

Human subjects: All authors have confirmed that this study did not involve human participants or tissue. Animal subjects: All authors have confirmed that this study did not involve animal subjects or tissue. Conflicts of interest: In compliance with the ICMJE uniform disclosure form, all authors declare the following: Payment/services info: All authors have declared that no financial support was received from any organization for the submitted work. Financial relationships: All authors have declared that they have no financial relationships at present or within the previous three years with any organizations that might have an interest in the submitted work. Other relationships: All authors have declared that there are no other relationships or activities that could appear to have influenced the submitted work.

\section{References}

1. Moynihan R: Key opinion leaders: independent experts or drug representatives in disguise? . BMJ. 2008, 336:1402-1403. 10.1136/bmj.39575.675787.651

2. Ross JS, Keyhani S, Korenstein D: Appropriateness of collaborations between industry and the medical profession: physicians' perceptions. Am J Med. 2009, 122:955-960. 10.1016/j.amjmed.2009.04.013

3. Fleischman W: Association between payments from manufacturers of pharmaceuticals to physicians and regional prescribing: cross sectional ecological study. BMJ. 2016, 354:i4189. 10.1136/bmj.i4189

4. Glass HE: Open Payments and the US clinical landscape. Ther Innov Regul Sci. 2019, 54:390-395. 10.1007/s43441-019-00067-5

5. Annapureddy A, Murugiah K, Minges KE, Chui PW, Desai N, Curtis JP: Industry payments to cardiologists. An analysis of the Open Payments Program from 2014 to 2016. Circ Cardiovasc Qual Outcomes. 2018, 11:10.1161/CIRCOUTCOMES.118.005016

6. Annapureddy A, Sengodan P, Mahajan S, Annapureddy T, Murugiah K, Desai NR, Curtis JP: Distribution of industry payments among medical directors of catheterization and electrophysiology laboratories from the top 100 US hospitals. JAMA Intern Med. 2019, 179:1282-1284. 10.1001/jamainternmed.2018.8775

7. Bailey CS, Fehlings MG, Rampersaud R, Hall H, Wai EK, Fisher CG: Industry and evidence-based medicine: believable or conflicted? A systematic review of the surgical literature. Can J Surg. 2011, 54:321-326. $10.1503 /$ cjs.008610

8. Chao AH, Gangopadhyay N: Industry financial relationships in plastic surgery: analysis of the Sunshine Act Open Payments database. Plast Reconstr Surg. 2016, 138:341-348. 10.1097/PRS.0000000000002404

9. Marszalek J, Chi G, Shojaei F, Yadala S, Veerapaneni K, Nalleballe K: Financial relationship between the industry and clinical neurophysiology: analysis of Open Payments data from 2013 to 2018. Neurology. 2020, 94:915.

10. Nalleballe K: Payments from industry to US vascular neurologists . PharmacoEcon Outcomes News. 2020, 848:20. 10.1007/s40274-020-6633-8

11. Narayanaswami P, Ahlawat A: Author response: financial relationships between neurologists and industry: the 2015 Open Payments database. Neurology. 2019, 92:351-352. 10.1212/WNL.0000000000006912

12. Nalleballe K, Sheng S, Li C, et al.: Industry payment to vascular neurologists. A 6-year analysis of the Open Payments Program from 2013 through 2018. Stroke. 2020, 51:1339-1343. 10.1161/STROKEAHA.119.027967

13. Sheng $\mathrm{S}$, Nalleballe $\mathrm{K}$, Brown $\mathrm{A}$, et al.: Industry payments to vascular neurologists, a six-year analysis of the Open Payments Program from 2013 to 2018. Stroke. 2020, 51:96.

14. Harada Y, Sheng S, Thombre V, et al.: A neuromuscular-based analysis of the Open Payments Program . Muscle Nerve. 2020, [Epub ahead of print]:10.1002/mus.27016 